\title{
Evaluación de la colposcopia en el estudio de los pacientes con neoplasia cervical
}

\author{
Franco Barros P.; Gildardo Gallego N.; Jaime Uribe D.; Jaime Botero U.; Elí Uechek M.*; Jairo Estrada R.**
}

RESUMEN: Se analizaron 409 historias clínicas de pacientes del HUSVP que consultaron entre enero de 1986 y diciembre de 1989, evaluadas por colposcopias por citología vaginal anormal, de éstas 367 casos con diagnóstico inicial de neoplasia cervical intraepital (NIC), a las cuales se les practicó histerectomía o conización y se pudo correlacionar el diagnóstico histológico de la biopsia inicial con el del espécimen quirúrgico, se revisaron para este trabajo.

El curetaje endocervical (cec) se realizó en todas las pacientes con colposcopia no satisfactoria y en algunos con colposcopia satisfactoria. El porcentaje de curetajes endocervicales positivos en los casos de colposcopias satisfactoria fue del $27.7 \%$. En ningún caso de colposcopia satisfactoria se encontró lesión invasora en el espécimen quirúrgico. La rata de curetaje endocervical positivo en los casos de colposcopia no satisfactoria fue de $\mathbf{4 2 . 1 0} \%$.

Los ocho casos de lesiones invasoras (seis invasoras y dos micros), en toda la serie, tenían colposcopia no satisfactoria y exceptuando un caso, todas tenían curetaje endocervical positivo. El porcentaje real de conizaciones diagnósticas es del 3.54\%. Se resalta la gran utilidad del curetaje endocervical cuando la colposcopia no es satisfactoria y de la conización diagnóstica, cuando la colposcopia no es satisfactoria y el curetaje endocervical es positivo.

PALABRAS CLAVES: Coldoscopia, neoplasia cervical intraepitelial (NIC).

SUMMARY: 409 medical records were analyzed corresponding to HUSVP patients who had consulted between January 1986 and December 1989, they were evaluated by colposcopy in reason of abnormal Pap Smears, 367 of these cases with an initial diagnosis of CIN, who had undergone hysterectomy or conization and it was possible to correlate the histological diagnosis of the initial biopsy with the surgical sample, were reviewed for this study.

The endocervical curetage (cec) was practiced on all patients with a non-satisfactory colposcopy and in some with a satisfactory colposcopy. The percentage of a positive endocervical curetages in the cases of satisfactory colposcopies was $27.7 \%$. No invasive lession was found in the surgical sample in any of the satisfactory colposcopy cases. The rate of positive endocervical curetage in the cases of non-satisfactory colposcopy was of $\mathbf{4 2 . 1 0} \%$.

The 8 cases of invasive lessions (6 invasive and 2 micros), in all the series, had non-satisfactory colposcopies, except for one case, they all had positive endocervical curetage. The real percentage of diagnostic conization is of $3.54 \%$. The great value of the endocervical curetage is highlighted, when the colposcopy is non-satisfactory and of the diagnostic conization, when the colposcopy is non-satisfactory and the endocervical curetage is positive.

KEY WORDS: Colposcopy, cervical intraephitelial neoplasia (CIN).

\section{Introducción}

En la actualidad uno de los métodos más importantes para evaluar las pacientes con citología vaginal anormal, es la colposcopia.

La citología vaginal detecta las pacientes con neoplasia cervical, especialmente aquellas sin lesión microscópica evidente, pero por la colposcopia podemos no sólo localizar la lesión, sino evaluar sus características y su extensión. No existe unanimidad en cuanto a la realización del curetaje endocervical, algunos investigadores consideran que se debe realizar rutinariamente en la evaluación colposcopica (1-5).

Otros no están de acuerdo con la realización del curetaje endocervical (CEC) de manera rutinaria, en todas las evaluaciones colposcópicas (6-9) ya que la omisión del CEC no disminuye su seguridad diagnóstica.

Este trabajo se realizó con los siguientes objetivos:

\footnotetext{
* Profesores Obstetricia y Ginecología Universidad de Antioquia.

** Ginecobstetra ISS-Especialista en entrenamiento en Oncología Ginecológica.
}

1. Determinar la seguridad diagnóstica de la colposcopia en el estudio de las pacientes con neoplasia cervical.

2. Determinar el valor del CEC en el estudio de estas pacientes.

3. Conocer el porcentaje de carcinomas invasores que se pasan por alto en la evaluación colposcópica.

\section{Material y métodos}

Se revisaron 409 historias clínicas de pacientes del Hospital Universitario San Vicente de Paúl (HUSVP) de Medellín, las cuales se evaluaron por colposcopia, por citología vaginal anormal, entre enero de 1986 y diciembre de 1989. De éstas en 28 casos el diagnóstico definitivo fue infección por Papiloma Virus. Tres pacientes con diagnóstico inicial de neoplasia cervical intraepitelial (NIC), fueron tratadas fuera de Medellín y no conocemos el resultado de la patología del espécimen quirúrgico. En otras tres pacientes por biopsia no dirigida por colposcopia, se diagnosticó NIC II en un caso y CA intraepitelial en los otros dos, pero al realizarles colposcopia y biopsia se comprobó lesión invasora. Cinco casos con colposcopia satisfactoria, con diagnóstico por biopsia de NIC I en tres casos y de NIC II en dos 
han seguido controlándose con citología y con colposcopia por más de seis meses. Tres pacientes se encontraban embarazadas, tenían citología vaginal anormal y NIC diagnosticado por biopsia por colposcopia.

Los otros 367 casos, en los cuales se practicó histerectomía o conización y se pudo correlacionar el diagnóstico histológico del espécimen quirúrgico con el diagnóstico de la biopsia por colposcopia, se analizaron para este trabajo.

Cuando en la colposcopia toda la lesión colposcópica y la unión escamocolumnar eran completamente visibles, ésta se consideraba colposcopia satisfactoria, si no llenaba estos criterios, se consideraba colposcopia no satisfactoria. El curetaje endocervical se consideró positivo cuando el informe de patología incluía una displasia o Ca intraepitelial y negativo cuando los fragmentos de epitelio columnar eran normales. El CEC se practicó en todas las pacientes con colposcopia no satisfactoria y sólo se hizo en 37 de las 309 con colposcopia satisfactoria. Se tomó como diagnóstico definitivo o final el diagnóstico histopatológico más severo en la biopsia inicial o en el cono, o en el espécimen de la histerectomía. Se consideró carcinoma microinvasor, cuando la profundidad de la invasión en el estroma es menor de tres milímetros desde la membrana basal y no habían células tumorales en los espacios linfáticos ni vasculares.

\section{Resultados}

Tabla 1

EVALUACION COLPOSCOPICA HUSVP 1986-1989

\begin{tabular}{|lrrr|}
\hline \multicolumn{2}{|c|}{$\begin{array}{l}\text { Colposcopias } \\
\text { Satisfactorias }\end{array}$} & \multicolumn{2}{c|}{ Colposcopias } \\
309 & $84 \%$ & No satisfactorias \\
\hline
\end{tabular}

El $16 \%$ de las pacientes tenía evaluaciones colposcópicas consideradas no satisfactorias y el $84 \%$ como satisfactorias, estas cifras son semejantes a las de Krebs (10) que encontró el $13.1 \%$ y el $86.9 \%$ respectivamente.

Tabla 2

EVALUACION COLPOSCOPICA CURETAJES ENDOCERVICALES

\begin{tabular}{|llll|}
\hline Colposcopias & $\begin{array}{l}\text { CEC } \\
\text { Positivos }\end{array}$ & $\begin{array}{l}\text { CEC } \\
\text { Negativos }\end{array}$ & Total \\
\hline Satisfactorias & $10(27.77 \%)$ & $26(72.23 \%)$ & 36 \\
Nosatisfactorias & $24(42.10 \%)$ & $33(57.90 \%)$ & 57 \\
\hline
\end{tabular}

Se realizaron 96 CEC, pero en dos casos de colposcopia satisfactoria el informe de patología fue de material insuficiente. En otro caso de colposcopia no satisfactoria, no se hizo CEC. En 36 colposcopias satisfactorias se realizó CEC siendo positivo en $10(27.77 \%)$, cuando la colposcopia no fue satisfactoria el CEC fue positivo en el $42.10 \%$.

De 309 casos con colposcopia satisfactoria, en 219 coincidió el diagnóstico inicial de NIC III con el de NIC III en el espécimen quirúrgico, lo que corresponde al $70.89 \%$ de exactitud; en 53 casos el diagnóstico inicial fue NIC III y en el espécimen quirúrgico (cono o útero) fue NIC II. En seis casos en los cuales la biopsia inicial informó NIC III
Tabla 3

COLPOSCOPIAS SATISFACTORIAS. Correlación entre la biopsia inicial y el diagnóstico en el espécimen quirúrgico.

\begin{tabular}{|c|c|c|c|c|c|c|c|}
\hline \multicolumn{8}{|c|}{ D\% Espécimen quirúrgico } \\
\hline B\% & Inic & NIC I & NIC II & NIC III & Micro & Invasor & Neg. \\
\hline NIC & I & & 1 & & & & 1 \\
\hline NIC & II & & 3 & 5 & & & 1 \\
\hline $\mathrm{NIC}$ & III & 20 & 53 & 219 & & & 6 \\
\hline
\end{tabular}

en el espécimen quirúrgico no se encontró neoplasia. En ningún caso se diagnosticó $\mathrm{Ca}$ invasor en los especímenes quirúrgicos.

Tabla 4

EVALUACION COLPOSCOPICA. HUSVP. 1986-1989. COLPOSCOPIAS NO SATISFACTORIAS. Correlación entre la biopsia inicial y el diagnóstico del espécimen quirúrgico.

\begin{tabular}{|llcccccc|}
\hline \multicolumn{7}{|c|}{ D\% } & Espécimen quirúrgico \\
\hline B \% & Inic & NIC I & NIC II & NIC III & Micro & Invasor & Neg. \\
\hline NIC & I & & 1 & 1 & & & \\
NIC & II & & 1 & 2 & & & \\
NIC & III & 2 & 3 & 38 & 2 & 6 & 2 \\
\hline
\end{tabular}

El total de colposcopias no satisfactorias fue de 58. En 38 casos, en los cuales en la biopsia inicial el diagnóstico fue NIC III, en el espécimen de la conización o el de la histerectomía fue el mismo.

En tres casos no se encontró lesión neoplásica en el espécimen quirúrgico, en ocho casos en los cuales la biopsia inicial informó NIC III, en dos el diagnóstico en el cono o en el útero fue $\mathrm{Ca}$ microinvasor y en seis $\mathrm{Ca}$ invasor.

Tabla 5

EVALUACION COLPOSCOPICA. HUSVP. 1986-1989. COLPOSCOPIAS NO SATISFACTORIAS. CEC NEGATIVOS. Correlación biopsia inicial y D\% definitivo.

\begin{tabular}{|c|c|c|c|c|c|c|}
\hline \multicolumn{7}{|c|}{ D\% definitivo } \\
\hline B $\%$ & Inic & NIC I & NIC II & NIC III & Micro & Invasor \\
\hline NIC & I & & & 1 & & \\
\hline NIC & II & & 1 & 1 & & \\
\hline NIC & III & 1 & 1 & 27 & & 1 \\
\hline
\end{tabular}

En 33 casos de colposcopias no satisfactorias el CEC fue negativo y de esas en 27 en las que el diagnóstico inicial fue NIC III, el diagnóstico definitivo fue igual, pero en un caso con diagnóstico inicial de Ca in situ, se encontró lesión invasora en el espécimen quirúrgico. A una de las pacientes con colposcopia no satisfactoria no se le hizo CEC.

Tabla 6

EVALUACION COLPOSCOPICA HUSVP. 1986-1989. Colposcopia no satisfactoria-CEC negativo. Cirugías-Patología Espécimen-Quirúrgicos.

\begin{tabular}{|lc|}
\hline Conización & Histerectomía \\
\hline NIC 10 & NIC 20 \\
Neg. 1 & Neg. 1 \\
& Inv. 1 \\
\hline
\end{tabular}


Se observa como en la tabla 6 que un caso de $\mathrm{Ca}$ invasor se pasó por alto en la evaluación colposcópica y que el diagnóstico se hizo en el espécimen de la histerectomía.

Tabla 7

EVALUACION COLPOSCOPICA HUSVP. 1986-1989. Colposcopias no satisfactorias CEC positivos. Correlación entre $\mathbf{B} \%$ inicial y D \% definitivo

\begin{tabular}{|lccccc|}
\hline \multicolumn{5}{|c|}{ Diagnóstico definitivo } \\
\hline $\begin{array}{l}\text { B } \% \\
\text { Inicial }\end{array}$ & NIC I & NIC II & NIC III & Micro & Invasor \\
\hline NIC I & & 1 & & & \\
NIC II & 1 & 1 & 13 & 2 & 5 \\
NIC III & 1 & & & & \\
\hline
\end{tabular}

En 13 casos con diagnóstico inicial NIC III, el diagnóstico definitivo fue igual, en dos casos que tenían diagnóstico inicial NIC III, el definitivo fue de cammicroinvasor y en cinco casos que tenían también diagnóstico inicial NIC III (cuatro de $\mathrm{Ca}$ in situ y uno de displasia severa) el diagnóstico final o definitivo fue de $\mathrm{Ca}$ invasor.

Tabla 8

EVALUACION COLPOSCOPICA HUSVP. 1986-1989. Colposcopias no satisfactorias -CEC positivos. Cirugía-Patología especimenes quirúrgicos

\begin{tabular}{|lllr|}
\hline Conización & & \multicolumn{3}{c|}{ Histerectomía } \\
\hline Nic & 7 & Nic & 10 \\
Micro & 1 & Micro & 1 \\
Invas. & 2 & Invas. & 3 \\
\hline
\end{tabular}

En los casos de colposcopias no satisfactorias con CEC positivo se practicaron 10 conizaciones diagnósticas y 14 histerectomías. En dos de las conizaciones el diagnóstico fue $\mathrm{Ca}$ invasor y en una $\mathrm{Ca}$ microinvasor. En los especímenes de la histerectomía, en tres casos se encontró Ca invasor que se había pasado por alto, además se encontró un Ca microinvasor. Se realizaron 10 conizaciones diagnósticas que corresponde al $2.72 \%$.

Tabla 9

EVALUACION COLPOSCOPICA HUSVP. 1986-1989. Análisis Carcinomas Invasores

\begin{tabular}{|cllccl|}
\hline Edad & Colposcop. & B $\%$ & CEC & Cono & Utero \\
\hline 60 & No satisf. & Ca In situ & Positivo & Ca invas. \\
34 & No satisf. & Ca In situ & Positivo & Ca invas. & \\
74 & No satisf. & Ca In situ & No & No & Ca invas. \\
56 & No satisf. & Ca In situ & Positivo & No & Ca invas. \\
40 & No satisf. & Displ. Sev. & Positivo & No & Ca invas. \\
50 & No satisf. & Ca In situ & Negativo & No & Ca invas. \\
46 & No satisf. & Ca In situ & Positivo & No & Micro \\
52 & No satisf. & Ca In situ & Positivo & Micro & \\
\hline
\end{tabular}

Como se observa en este cuadro el diagnóstico definitivo fue de carcinoma microinvasor en dos casos y de $\mathrm{Ca}$ invasor en seis. En todos estos casos la colposcopia fue no satisfactoria y la biopsia inicial fue informada como NIC III. En una de estas pacientes no se realizó CEC, ni conización, pasándose por alto el $\mathrm{Ca}$ invasor.

En otro caso el CEC fue informado como negativo, no se le hizo conización y en el espécimen de la histerectomía, se encontró $\mathrm{Ca}$ invasor.

De tres casos con CEC positivo que se sometieron a conización, se diagnosticó $\mathrm{Ca}$ invasor en dos y en una $\mathrm{Ca}$ microinvasor. En otros tres casos con CEC positivo no se hizo conización, se pasó directamente a la histerectomía y el informe de patología del útero fue $\mathrm{Ca}$ invasor en dos casos y de microinvasor en uno.

\section{Comentarios}

Desde 1979 contamos con la colposcopia para la evaluación de las pacientes con citología vaginal anormal en el grupo de oncología ginecológica del HUSVP. En una revisión realizada en 1980 (11), antes de contar con la colposcopia, se encontró una falla en el diagnóstico de $\mathrm{Ca}$ invasor del $5.03 \%$.

Algunos investigadores (12) sugieren que el error más común en la colposcopia es la omisión del CEC, otros $(1$, $3,4)$ también están de acuerdo que en el CEC se realice de manera rutinaria. Sin embargo,Talebian (13) no adicionaba el CEC a la biopsia cervical cuando la colposcopia era satisfactoria y reportó el $100 \%$ de seguridad diagnóstica. Swan (9) sin realizar curetaje endocervical, obtuvo una seguridad diagnóstica del $98.4 \%$ comparable a otros reportes de la literatura que utilizaban la biopsia cervical más el CEC y la rata de conizaciones diagnósticas fue del $7 \%$. Javaheri (6) concluyó que la omisión del CEC cuando la colposcopia es satisfactoria no disminuye su seguridad diagnóstica. Urcuyo (8) encontró que el CEC fue innecesario en las colposcopias satisfactorias, pero que si puede ayudar a evitar pasar por alto el $\mathrm{Ca}$ invasor en la colposcopia no satisfactoria.

El porcentaje de CEC positivos en casos de colposcopias satisfactorias en este trabajo fue del $27.77 \%$., cifra superior al $17.0 \%$ de Drescher (4) y al $8.6 \%$ de Urcuyo (8). Spirtos (13) encontró que en 20 de 210 pacientes con colposcopia satisfactoria, el CEC era potencialmente contaminado, como se deduce por el desgarro producido, en la lesión en el exocervx, al examen colposcópico después de realizar el CEC.

La rata de CEC positivo en pacientes con colposcopia no satisfactoria fue del $42.10 \%$, cifra semejante a la de Drescher (4) del $48.4 \%$ y a la de Moseley (14) del $45 \%$ pero inferior a la de Urcuyo (8) que encontró CEC positivo en colposcopia no satisfactoria en el 57.3\% y Hatch (1) en el $52.7 \%$.

De 24 pacientes con colposcopia no satisfactoria y CEC positivo en siete se hizo diagnóstico final de $\mathrm{Ca}$ invasor o microinvasor, o sea, el $29.1 \%$, Hatch (1) encontró una cifra inferior: 14 lesiones invasoras en 125 colposcopias no satisfactorias con CEC positivos: $11.2 \%$. Moseley (14) encontró seis lesiones invasoras en 51 casos de colposcopia no satisfactoria y CEC positivo o sea el $11.8 \%$.

En 219 de 309 casos de colposcopia satisfactoria, el diagnóstico de la biopsia dirigida y el del espécimen quirúrgico fue el mismo, en ningún caso se pasó por alto el $\mathrm{Ca}$ invasor. Sólo en 36 pacientes con colposcopia satisfactoria, inicialmente se realizó CEC, pero posteriormente de acuerdo con la literatura, se decidió no realizarlo de manera rutinaria 
en estos casos. Hatch informó de tres Ca invasores en 1819 colposcopias satisfactorias que corresponden al $0.16 \%$. Generalmente en los casos de colposcopia satisfactoria se presenta lesión invasora, el diagnóstico se hace en la biopsia inicial. En la tabla 3 se observa que cuando la colposcopia es satisfactoria en ningún caso se pasó por alto el diagnóstico de lesión invasora.

De 33 casos con colposcopia no satisfactoria y CEC negativo, en uno se encontró $\mathrm{Ca}$ invasor en el espécimen de la histerectomía.

De 33 casos con colposcopia no satisfactoria y CEC negativo, en uno se encontró $\mathrm{Ca}$ invasor en el espécimen de la histerectomía.

Urcuyo (9) informó de otro caso invasor en una colposcopia no satisfactoria y CEC negativo en 117 o sea en el $0.85 \%$. Otros $(3,15)$ han informado CEC negativo en casos de diagnóstico de $\mathrm{Ca}$ invasor en el $2.73 \%$ y en el $0.79 \%$ respectivamente, pero sin detallar si se trataba de colposcopia satisfactoria o no.

Townsend (12) informó de seis lesiones invasoras (tres micro y tres invasoras) en 50 casos que tenían CEC positivo, pero no informan si se trataba de colposcopia satisfactoria. Otros (16) informan el $12 \%$ de lesiones invasoras cuando el CEC fue positivo. Sin embargo algunos (1-3) están de acuerdo en que el CEC negativo descarta un $\mathrm{Ca}$ invasor. Se puede observar, como lo mencionan algunos $(4,10)$ que la combinación de CEC positivo y colposcopia no satisfactoria identifica un grupo de pacientes con riesgo aumentado de tener un $\mathrm{Ca}$ invasor.

Se realizaron 10 conizaciones diagnósticas (Tabla 8) en pacientes con colposcopias no satisfactorias y CEC positivo, lo que corresponde al $2.7 \%$ del total, cifra semejante a la de otros $(1,6)$ del 3.1 y $3 \%$.

$\mathrm{Al}$ revisar los casos con diagnósticos definitivo o final de $\mathrm{Ca}$ microinvasor o invasor en toda la serie se encontraron ocho lo que corresponde al $2.17 \%$ del total, cifra semejante a la de Drescher (4) del $2.2 \%$ en una serie de 540 pacientes. En la tabla 9 se observa que en todos los casos de lesiones invasoras las colposcopias eran no satisfactorias. En tres con CEC positivo se hizo conización diagnóstica y se comprobó lesión invasora. En otros tres casos con CEC positivo no se hizo conización diagnóstica, se les realizó histerectomía, encontrándose lesión invasora. En un caso el CEC fue negativo, no se le hizo conización, se le practicó histerectomía diagnosticándose $\mathrm{Ca}$ invasor en el espécimen quirúrgico. En otra paciente no se practicó CEC (se desconoce la razón), se realizó histerectomía y se encontró $\mathrm{Ca}$ invasor.

Aun cuando se dice (17) que la colposcopia elimina en un $95 \%$ la necesidad de conización diagnóstica, como se ha visto en todo lo expuesto anteriormente, cuando la colposcopia no es satisfactoria y el CEC es positivo, se debe realizar conización diagnóstica. En uno de los casos de $\mathrm{Ca}$ invasor, con colposcopia no satisfactoria el CEC fue negativo, sin embargo cuando el CEC es negativo es muy poco probable que exista un $\mathrm{Ca}$ invasor.

Por esto se dice (1) que el curetaje endocervical ha permitido una reducción en el número de conizaciones diagnósticas en pacientes con colposcopia no satisfactoria.

De manera que a los 10 casos de conización diagnóstica, se deben agregar los tres que se omitieron teniendo CEC positivo con colposcopia no satisfactoria y así la rata de conos diagnósticos verdadera, sería del $3.54 \%$.

Teniendo en cuenta que en una de las pacientes con $\mathrm{Ca}$ invasor, con colposcopia no satisfactoria estaba indicando el curetaje endocervical y éste no se realizó y que en otro el CEC fue negativo, podemos concluir que en la serie de 367 casos, se pasó por alto el Ca invasor como falla propiamente dicha del método de la evaluación colposcópica, en un solo caso.

\section{BIBLIOGRAFIA}

1. Hatch KD., Shingleton HM., Orr JW. et al. Role of Endocervical Curettaje in Colposcopy. Obstet. Gynecol. 1985; 65: 403.

2. Townsend DE., Ostergard DR., Mishell DR. et al. Abnormal papanicolau Smears. Am. J. Obstet. Gynecol 1970; 108: 429.

3. Shingleton HM., Gore H., Austin JM. Out Patient Evaluation of Patients with Atipical Papanicolau Smears: Contribution of Endocervical Curettage. Am. J. Obstet. Gynecol. 1976; 126: 122.

4. Drescher CW., Peters WA., Roberts JA. Contribution of Endocervical Curettage in Evaluatioting Obnormal Cervical Cytology Obstet. Gynecol. 1983; 62: 343.

5. Shingleton HM., Patridge EE., Austin JM. The Significance of Age in the Colposcopic Evaluation of Women with Atipical Papanicolau Swears Obstet. Gynecol. 1977; 49: 61.

6. Javaheri G., Fedgin MD. Diagnostic Value of Colposcopi in the Investigation of Cervical Neoplasia. Am. J. Obstet. Gynecol. 1980; 137: 588

7. Talebian F., Shayana., Krumholz BA. et al. Colposcopi Evaluation of Patients with Abnormal Cervical Cytology Obstet. Gynecol. 1977; 49: 670 .

8. Urcuyo R., Rome RM., Nelson JH. Some Observations on the Value of Endocervical Curettage Performed as an Integral Part of Colposcopic Examination of Patients with Abnormal Cervical Cytology Am. J. Obstet. Gynecol. 1977: 128: 787.
9. Swan RW. Evaluation of Colposcopic Occupacy Without Endocervical Curettage Obstet. Gynecol. 1979; 53: 680.

10. Krebs HB., Wheelock JB., Hurt WG. Positive Endocervical Curettage in Patients with Satisfactory and Insatisfactory Colposcopy: Clinical Implications Obstet. Gynecol. 1987; 69: 601.

11. Barros PF., Gallego NG. Carcinoma Cervical Intraepitelial. Rev Col. Obstet. Gynecol. 1980; 31: 50.

12. Townsend DE., Richard RM., Marks E. et al. Invasive Cancer Following out Patient Evaluation and Therapy for Cervical Disease Obstet. Gynecol. 1981; 57: 145.

13. Spirtos NM., Schlaerth JB., Ablaing G. et al. A Critical Evaluation of the Endocervical Curettage Obstet. Gynecol. 1987; 70: 729.

14. Moseley KR., Dinh TV., Hannigan EV. et al. Necessity of Endocervical Curettage in Colposcopy. Am J. Obstet. Gynecol. 1986; 154: 992.

15. Jafari K., Sansguiri R. Role of Endocervical Curettage in Colposcopy. Am. J. Obstet. Gynecol. 1978; 131: 183.

16. Tredway DR., Townsen DE., Hovland DN. et al. Colposcopy and Crio Surgery in Cervical Intraephitelial Neoplasia. Am. J. Obstet. Gynecol. 1972; 114: 1020 .

17. Morrow CP., Townsend DE. Synopsis of Gynecologic Oncology Third Edition. New York John Wiley and Sons, 1987. 